\title{
ON THE EXISTENCE OF SHILOV BOUNDARIES
}

\author{
RAINER WITTMANN
}

\begin{abstract}
The existence of a carrier in the sense of potential theory is proved for rather general cones of functions. The existence of the Shilov boundary is an immediate consequence of this result.
\end{abstract}

1. The main results. Let $X$ be a compact space and $E \subset X$ such that $\bar{E}=X$. Let $\mathscr{F}$ be a set of lower bounded numerical functions on $E$. We assume that there exists a convex cone $\mathcal{S} \subset \mathcal{F}$ which possesses the following properties:

(S1) $u+s \in \mathscr{F}$ for any $u \in \mathscr{F}, s \in \mathcal{S}$.

( 2 ) For any $s \in \mathcal{S}$, there exists a continuous extension $\bar{s}$ to $X$ (which is obviously unique).

(ऽ3) There exists a function $s \in \mathcal{S}$ such that $\bar{s}$ is strictly positive on $X$.

(ऽ4) For any $x, y \in X, x \neq y$, there exists $s, t \in \mathcal{S}$ such that $\bar{s}(x) \cdot \bar{t}(y) \neq$ $\bar{s}(y) \cdot \bar{t}(x)$.

A closed set $K \subset X$ is called a carrier set of a numerical function $f$ on $E$ iff, for any open $V \subset X, K \subset V$ and any $u \in \mathscr{F}, f \leqslant u$ on $V \cap E$ implies $f \leqslant u$ throughout $E$. Obviously $\overline{\{f>0\}}$ is a carrier set of $f$ if $\mathscr{F}$ is a cone of nonnegative functions. For any numerical function $f$ on $E$, we denote by $\operatorname{carr}(f)$ the smallest carrier set of $f$ if it exists. carr $(f)$ is called the carrier of $f$ (with respect to $\mathscr{F}$ ).

1.1. THEOREM. For every upper bounded numerical function $f$ on $E, \operatorname{carr}(f)$ exists.

Proof. We denote

$$
\tilde{\mathscr{F}}:=\{\inf \mathcal{G}: \mathcal{G} \subset \mathscr{F} \text { finite }\} \text { and } \tilde{\mathcal{S}}:=\{\inf \mathcal{G}: \mathcal{G} \subset \mathcal{S} \text { finite }\} .
$$

It is easy to see that $\tilde{\mathscr{F}}$ and $\tilde{\mathcal{S}}$ satisfy again the conditions $(\delta 1)-(\delta 4)$ and that the carrier sets of $f$ with respect to $\mathscr{F}$ are exactly the carrier sets of $f$ with respect to $\tilde{\mathcal{F}}$. Hence we may assume that the convex cone $\mathcal{S}$ is inf-stable.

Let $K_{1}, K_{2}$ be two carrier sets of $f$, let $V \subset X$ be open such that $K_{1} \cap K_{2} \subset V$ and let $u \in \mathscr{F}$ be such that $1_{V \cap E} \cdot f \leqslant u$.

Since $K_{1}^{\prime}:=K_{1} \backslash V$ and $K_{2}$ are disjoint, there exist open sets $V_{1}^{\prime}, V_{2}$ such that $K_{1}^{\prime} \subset V_{1}^{\prime}, K_{2} \subset V_{2}$ and $\bar{V}_{1}^{\prime} \cap \bar{V}_{2}=\varnothing . \mathcal{H}:=\{\bar{s}-\bar{t}: s, t \in \mathcal{S}\}$ is a vector lattice of continuous functions on $X$. Hence, by the Stone-Weierstrass theorem, there exists $g \in \mathcal{H}$ such that $g \geqslant 1$ on $\bar{V}_{1}^{\prime}$ and $g \leqslant-1$ on $\bar{V}_{2}$. Thus there exist $s, t \in \mathcal{S}$ such that

Received by the editors June 21, 1982 and, in revised form, December 10, 1982.

1980 Mathematics Subject Classification. Primary 46A55.

Key words and phrases. Shilov boundary, carrier theory. 
$\bar{s}-\bar{t}=\max (0, g)$. Using $(\S 3)$, we may assume $s \geqslant 0, f \leqslant u+s$. By the construction of $s$ and $t$, there exist $0<\alpha<1$ such that

$$
t \leqslant \alpha \cdot s \text { on } V_{1}^{\prime} \cap E, \quad s=t \quad \text { on } V_{2} \cap E .
$$

For any $\beta \geqslant 0$ such that $f \leqslant u+\beta \cdot s$, we have $f \leqslant u+\beta \cdot t$ on $V_{2} \cap E$ and therefore, since $K_{2}$ is a carrier set,

$$
f \leqslant u+\beta \cdot t \leqslant u+\beta \cdot \alpha \cdot s \text { on } V_{1}^{\prime} \cap E .
$$

Thus we have $f \leqslant u+\beta \cdot \alpha \cdot s$ on $V_{1} \cap E$, where $V_{1}:=V \cup V_{1}^{\prime} \supset K_{1}$. Since $K_{1}$ is a carrier set, we get $f \leqslant u+\beta \cdot \alpha \cdot s$ on $E$. Starting with $\beta=1$, we get by iteration $f \leqslant u+\alpha^{n} \cdot s$ for any $n \in \mathbf{N}$ and hence $f \leqslant u$. We have shown that $K_{1} \cap K_{2}$ is a carrier set of $f$. Thus the set $C(f):=\{K \subset X: K$ carrier set of $f\}$ is stable with respect to finite intersections. Hence, for any open $V \supset C:=\bigcap_{K \in C(f)} K$, there exists $K \in C(f)$ such that $K \subset V$. This implies that $C$ is also a carrier set of $f$. Obviously $C$ is the smallest carrier set of $f$.

1.2. REMARK. The existence of a carrier in the above sense is basic for the construction of kernels in potential theory (see [4,3.4], [5, 8.1] and [2]). The above theorem applies in many of these situations. Especially the results of [4], 5.5 may be improved and complemented by the above theorem. This will be done in a subsequent paper. The applications to potential theory are the reason why we have formulated Theorem 1.1 in the above form. In the rest of the paper we apply Theorem 1.1 to existence theorems of the Shilov boundary.

A closed set $K \subset X$ is called a Shilov set (with respect to $\mathscr{F}$ ), iff, for any $u \in \mathcal{F}$, $\liminf _{E \ni y \rightarrow x} u(y) \geqslant 0$ for any $x \in K$, implies $u \geqslant 0$ on $E$.

The proof of the following simple lemma is left to the reader.

1.3. Lemma. The Shilov sets are exactly the carrier sets of the function 0.

1.4. Corollary. There exists a smallest Shilov set $\operatorname{Sh}(\mathcal{F})$ which is called the Shilov boundary.

1.5. REMARK. For point-separating vector spaces of continuous functions on $X$, the above corollary was shown by Arens and Singer in [1] and by Milman in [7]. Their result was generalized by Bauer to point-separating sets of lower semicontinuous functions which are stable with respect to addition. Bauer's result is not a special case of Corollary 1.3 and when certain additional conditions are imposed it even implies Corollary 1.3. However, Bauer's result depends on one hand on the HahnBanach theorem and on the other hand on Bauer's minimum principle which itself depends on Zorn's lemma.

\section{Application to function algebras.}

2.1. THEOREM. Let $Q$ be an algebra of bounded complex-valued functions on a compact space $X$ containing the function 1 . We assume that there exists a point-separating subalgebra $\mathscr{B}$ of continuous functions. Then there exists a smallest closed subset $\partial_{\mathscr{Q}} X \subset X$ possessing the following property.

For any $f \in \mathbb{Q}$, there exists $x \in \partial_{\mathscr{Q}} X$ such that

$$
\sup \{|f(y)|: y \in X\}=\underset{y \rightarrow x}{\limsup }|f(y)| \text {. }
$$


Proof. We consider $\mathscr{F}:=\{\alpha-\log |f|: \alpha \in \mathbf{R}, f \in \mathbb{Q}\}$ and $\delta:=\{\alpha-\log |f|:$ $\alpha \in \mathbf{R}, f \in \mathscr{T}\}$. Obviously $\mathscr{F}$ and $S$ satisfy conditions $(\delta 1)-(\delta 4)$, but $\delta$ is in general not a convex cone. Nevertheless the next lemma shows that we can apply Corollary 1.4 since we obviously have $u+v \in \mathscr{F}$ for any $u, v \in \mathscr{F}$.

2.2. LemMA. Let $\mathscr{F}$ be a set of lower bounded numerical functions on $X$ such that $u+v \in \mathcal{F}$ for any $u, v \in \mathcal{F}$ and $1 \in \mathcal{F}$. Let $\tilde{\mathcal{F}}$ be the convex cone generated by $\mathscr{F}$. Then a closed set $K \subset X$ is a Shilov set with respect to $\mathscr{F}$ iff it is a Shilov set with respect to $\tilde{\mathscr{F}}$.

Proof. We denote $\mathcal{F}^{\prime}:=\left\{q \cdot u: q \in \mathbf{Q}_{+}, u \in \mathscr{F}\right\}$. We claim that $q_{1} \cdot u_{1}+q_{2} \cdot u_{2}$ $\in \mathcal{F}^{\prime}$ for any $u_{1}, u_{2} \in \mathcal{F}^{\prime}$ and $q_{1}, q_{2} \in \mathbf{Q}_{+}$. Indeed, there exists $n \in \mathbf{N}$ such that $n \cdot q_{1} \cdot u_{1} \in \mathscr{F}, n \cdot q_{2} \cdot u_{2} \in \mathscr{F}$. Thus we have $n \cdot\left(q_{1} \cdot u_{1}+q_{2} \cdot u_{2}\right) \in \mathscr{F}$ and therefore $\left(q_{1} \cdot u_{1}+q_{2} \cdot u_{2}\right) \in \mathscr{F}^{\prime}$. Now let $K$ be a Shilov set with respect to $\mathscr{F}$ and let $u \in \tilde{\mathscr{F}}$ be such that $\liminf _{y \rightarrow x} u(y) \geqslant 0$ for any $x \in K$. There exist $\alpha_{i} \in \mathbf{R}_{+}$and $v_{i} \in \mathscr{F}$ such that $\sum_{i=1}^{n} \alpha_{i} v_{i}=u$. Since the functions $v_{i}$ are lower bounded for any $\varepsilon>0$ there exist $\alpha_{i}^{\prime} \in \mathbf{Q}$ such that $u+\varepsilon \geqslant u^{\prime}:=\sum_{i=1}^{n} \alpha_{i}^{\prime} v_{i}$ and $\liminf _{y \rightarrow x} u^{\prime}(y)+\varepsilon \geqslant 0$. Since $K$ is obviously also a Shilov set with respect to $F^{\prime}$ and $u^{\prime}+\varepsilon \in \mathscr{F}^{\prime}$ for $\varepsilon \in \mathbf{Q}$, we get $u+2 \varepsilon \geqslant u^{\prime}+\varepsilon \geqslant 0$. Hence $K$ is a Shilov set with respect to $\tilde{\mathscr{F}}$. The converse implication is trivial.

2.3. REMARK. We should remark that Theorem 2.1 may also be shown by modifying the proof of [6, Theorem 3.1.18].

\section{REFERENCES}

1. R. Arens and I. M. Singer, Function values as boundary integrals, Proc. Amer. Math. Soc. 5 (1954), 735-745.

2. J. Azema, Noyau potentiel associé a une fonction excessive d'un processus de Markov, Ann. Inst. Fourier (Grenoble) 19 (2) (1969), 495-526.

3. H. Bauer, Šlovscher Rand und Dirichletsches Problem, Ann. Inst. Fourier (Grenoble) 11 (1961), $89-136$.

4. N. Boboc, Gh. Bucur and A. Cornea, Order and convexity in potential theory: H-cones, Lecture Notes in Math., vol. 853, Springer-Verlag, Berlin-Heidelberg-New York, 1981.

5. C. Constantinescu and A. Cornea, Potential theory on harmonic spaces, Springer-Verlag, BerlinHeidelberg-New York, 1972.

6. L. Hörmander, An introduction to complex analysis in several variables, North-Holland, AmsterdamLondon, 1973.

7. D. P. Milman, Characteristics of extremal points of regularly compact sets, Dokl. Akad. Nauk SSSR N. S. 57 (1947), 119-122. (Russian)

Katholische Universität Eichstätt, Mathematisch-Geographische Fakultät, Ostenstrasse 26-28, D-8078 EichSTÄTt, Federal Republic of Germany 